\title{
Generation of homoplasmic plastid transformants of a commercial cultivar of potato (Solanum tuberosum L.)
}

\author{
Thanh Thi Nguyen ${ }^{\mathrm{a}}$, Gregory Nugent ${ }^{\mathrm{a}, 1}$, Teodoro Cardi ${ }^{\mathrm{a}, \mathrm{b}}$, Philip John Dix ${ }^{\mathrm{a}, *}$

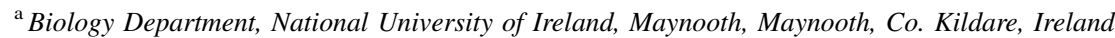 \\ ${ }^{\mathrm{b}}$ CNR-IGV, Institute of Plant Genetics, Res. Div. Portici, Via Universitá 133, 80055 Portici, Italy
}

Received 25 August 2004; accepted 31 January 2005

Available online 20 March 2005

\begin{abstract}
This report describes the integration and expression of foreign genes into the plastid genome of a commercial cultivar of potato. Plastid transformation of potato was achieved using two tobacco specific plastid transformation vectors, pZS197 (Prrn/aadA/psbA3') and pMSK18

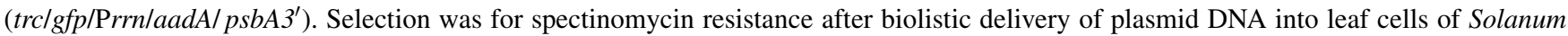
tuberosum cv. Desiree. Ten transplastomic lines were obtained from 179 bombarded samples with vector pZS197 and four transplastomic lines selected out of 103 bombarded samples with vector pMSK18. Southern blot and PCR analyses confirmed homoplasmy in the primary regenerants, and incorporation of the $a a d A$ and $g f p$ genes into the potato plastid genome by two homologous recombination events via the flanking plastid DNA sequences. Fluorometric measurements confirmed GFP expression in leaves and tubers of pMSK18 lines. No transformants were obtained with a third tobacco vector, pNtcZ7 (Prrn/gfp/psbA3'/trc/aadA/rrnB-ter) in which the selectable marker gene is driven by a bacterial $(t r c)$ promoter, which does permit selection of plastid transformants in tobacco, and allows low level expression of the reporter gene, $g f p$, in potato.
\end{abstract}

(C) 2005 Elsevier Ireland Ltd. All rights reserved.

Keywords: Plastid transformation; Chimeric aadA gene; gfp gene; Particle bombardment; Transplastomic potato

\section{Introduction}

Plastid transformation in higher plants offers some advantages over nuclear transformation, including maternal inheritance of transgenes, no position effects, as the genes of interest are introduced into the plastome via homologous recombination, and high level of foreign protein expression [1-3].

Plastid transformation in higher plants was first successfully carried out in tobacco using a mutant plastid $16 \mathrm{~S}$ ribosomal RNA gene for selection [4], but it is most

Abbreviations: CIM, callus induction medium; $\mathrm{GA}_{3}$, gibberelic acid; IAA, indole-3-acetic acid; MS, Murashige and Skoog (1962); SIM, shoot induction medium

* Corresponding author. Tel.: +353 1 7083836; fax: +3531 1083845 .

E-mail address: phil.dix @may.ie (P.J. Dix).

${ }^{1}$ Present address: Plant Biotechnology Centre, Primary Industries Research Victoria, La Trobe University, Bundoora, Vic., Australia. commonly reported with vectors containing a chimeric bacterial aadA gene, which confers resistance to spectinomycin and streptomycin [5]. Chloroplast transformation is a routine procedure only for tobacco, and extending it to other species is most important if the potential of the plastid as a production platform for large amounts of recombinant protein is to be realised. Plastid transformation has now been reported in Arabidopsis thaliana [6], potato [7], rice [8], tomato [9], Brassica napus [10] and Lesquerella fendleri [11]. Biolistics have been used for DNA delivery in all these studies. However, transformation efficiencies are at least 10 times lower than reported for tobacco. It can also be difficult to achieve homoplasmic transformants, where all plastid genome copies contain the introduced genes [8].

Plastid transformation in potato was first described by Sidorov et al. [7] using a breeding line, and this remains the sole report. In the current report we describe the reproducible plastid transformation of an important commercial 
potato cultivar, Solanum tuberosum cv. Desiree, and demonstrate the importance of a strong plastid promoter to obtain transformants.

\section{Materials and methods}

\subsection{Plastid transformation vectors}

Plasmid pZS197 (Prrn/aadA/T psbA3') was constructed for high frequency plastid transformation in tobacco [5]. The chimeric aadA gene is under the control of the ribosomal RNA operon promoter (Prrn) and the $3^{\prime}$ untranslated region (UTR) of the plastid psbA gene and was cloned between the plastid $r b c L$ and $a c c D$ genes for targeting to the large single copy (LSC) region of chloroplast genome. Plasmids pMSK18 (trc/gfp/Prrn/aadA/psbA3'-UTR) and pNtcZ7 (Prrn/gfp/psbA3'-UTR/trc/aadA/rrnB-ter) were also designed for chloroplast transformation in tobacco [12]. They both insert between the coding regions for $16 \mathrm{~S}$ rRNA and orf70B in the inverted repeat region. In pMSK18, the $g f p$ coding region is under the control of the bacterial trc promoter, while Prrn drives expression of the selectable marker gene aadA. In pNtcZ7 this situation is reversed.

\subsection{Plastid transformation procedure}

Solanum tuberosum cv. Desiree was obtained from the Department of Agriculture, Food and Rural Development, Ireland. Stock shoot cultures were grown in vitro in Magenta G7 (Sigma) containers on growth regulator free Murashige and Skoog [13] (MS) medium with Gamborg's B5 vitamins [14] containing $30 \mathrm{~g} / \mathrm{L}$ sucrose, and solidified with $0.8 \%$ (w/ v) agar. Media were adjusted to $\mathrm{pH} 5.7$ and autoclaved for 20 min at $121{ }^{\circ} \mathrm{C}$. Shoot cultures were grown at $22{ }^{\circ} \mathrm{C}$ under a $16 \mathrm{~h} \mathrm{light} / 8 \mathrm{~h}$ dark light regime. Three to four weeks after each subculture, four to five dark green leaves per rooted plant were harvested and the upper nodes were subcultured on MS medium as described above. Bombardment: two to four leaves were placed adaxial side up onto callus induction medium (CIM) containing MS salts with B5 vitamins (Duchefa), $2.0 \mathrm{mg} / \mathrm{L}$ 2,4-dichlorophenoxyacetic acid (2,4D), $0.9 \mathrm{mg} / \mathrm{L}$ zeatin riboside (ZR), $16 \mathrm{~g} / \mathrm{L}$ glucose [15] for $24 \mathrm{~h}$ before bombardment. Leaves were bombarded with gold particles $(0.6 \mu \mathrm{m}$ diameter) coated with plasmid DNA using a PDS 1000/He Biolistic gene gun (BioRad). A rupture disc pressure of $1100 \mathrm{psi}$, partial vacuum pressure between 25 and $28 \mathrm{in} \mathrm{Hg}$ and a target distance of $6 \mathrm{~cm}$ was used for bombardment. Two to three days after bombardment the leaves were cut into $3 \mathrm{~mm} \times 3 \mathrm{~mm}$ pieces and placed on CIM containing $300 \mathrm{mg} / \mathrm{L}$ spectinomycin and incubated under dim light in a $16 \mathrm{~h}$ light $/ 8 \mathrm{~h}$ dark regime for 4 weeks. The leaf explants were transferred to shoot induction medium (SIM) containing MS salts with B5 vitamins, $3.0 \mathrm{mg} / \mathrm{L} \mathrm{ZR}, 2.0 \mathrm{mg} / \mathrm{L}$ indole acetic acid (IAA), $1.0 \mathrm{mg} / \mathrm{L}$ gibberellic acid $\left(\mathrm{GA}_{3}\right), 16 \mathrm{~g} / \mathrm{L}$ glucose and $300 \mathrm{mg} / \mathrm{L}$ spectinomycin and subcultured to the same selective medium at 3-weekly intervals. Spectinomycin resistant shoots obtained in $8-10$ weeks were identified as green shoots on bleached leaf explants. Shoots were subcultured to growth regulator-free MS medium (RM) with $400 \mathrm{mg} / \mathrm{L}$ spectinomycin, for root formation. Rooted plants were transferred to soil in pots and grown to maturity in a growth room with the same temperature and daylength settings as used for the in vitro culture. Leaf explants and tuber pieces from putative transformants were tested on SIM medium for resistance to both spectinomycin $(300 \mathrm{mg} / \mathrm{L})$ and streptomycin $(300 \mathrm{mg} / \mathrm{L})$.

\subsection{PCR and Southern blot analyses of total cellular DNA}

Total leaf cellular DNA was extracted from plants using a method described previously [16]. Polymerase chain reactions (PCRs) were carried out using primers specific for the chimeric aadA or $g f p$ genes and analysis for homoplasmy used a pair of primers (RBCL/ACCD) or (pSSH-rev/trnV-Solanum) flanking the transgene insertion site in the potato chloroplast genome. Primers used for screening transformants and probe synthesis are as follows:

\begin{tabular}{ll}
\hline Oligo name & Sequence $\left(5^{\prime}-3^{\prime}\right)$ \\
\hline RBCL & CAGAGACTAAAGCAAGTGTTG \\
ACCD & CATGTCTTCATCCATAGGA \\
pSSH-rev & TCTTGATCAATCCCTTTGCCCTC \\
trnV Solanum & CATGTCTTCCATCCATAGGA \\
RB197F & GTCTACTTCTTCACATCCACC \\
RB197R & TCCATACTTCACAAGCAGC \\
\hline
\end{tabular}

PCR was performed with the AccuTaq kit (Sigma). The PCRs were run as follows: denaturing $94{ }^{\circ} \mathrm{C}, 30 \mathrm{~s}$, annealing 51-63 ${ }^{\circ} \mathrm{C}$ (depending on the Tm) $15 \mathrm{~s}$ and extension $68^{\circ} \mathrm{C}$ 4-6 min; for 30 cycles.

For Southern blot analysis, 5-7 $\mu \mathrm{g}$ of total DNA were digested with $E c o$ RI and EcoRV, separated in a $0.8 \%(w / v)$ agarose gel $(16 \mathrm{~h}, 25-30 \mathrm{~V})$ and transferred to a nylon membrane (Hybond $\mathrm{N}^{+}$; Amersham, Dublin, Ireland). Signal detection was performed using a non-radioactive DNA labeling [17] and detection protocol (DIG Probe Synthesis Kit, Roche, Mannheim, Germany). Plasmid pZS197 was used as template for synthesis of the probe using RB197F and RB197R primers. Digoxigenin-labeled PCR product was used for membrane hybridization. Immunological detection was performed using a chemiluminescent substrate (CDP-star, Roche). Signals were visualized by exposure to Kodak X-ray film for 1-5 min.

\subsection{Detection of GFP in transplastomic potato}

Total soluble protein was extracted from transplastomic lines in a buffer containing $0.1 \mathrm{M} \mathrm{Na}_{2} \mathrm{CO}_{3}$, pH 9.6 [18]. The protein concentration was determined using a BioRad protein assay kit. Potato leaf or tuber samples were 
Table 1

Results of chloroplast transformation experiments in potato

\begin{tabular}{lcccc}
\hline Plasmid & Shots & Spec $^{\mathrm{r}}$ shoots & $\begin{array}{l}\mathrm{Spec}^{\mathrm{r}} \text { and } \\
\text { strep }^{\mathrm{r}} \text { shoots }\end{array}$ & PCR positive \\
\hline pZS197 & 179 & 21 & 10 & 10 \\
pMSK18 & 103 & 9 & 4 & 4 \\
pNtcZ7 & 153 & 8 & 0 & 0 \\
\hline
\end{tabular}

equilibrated to a protein concentration of $1000 \mu \mathrm{g} / \mathrm{ml}$. Recombinant GFP [rGFP ${ }_{\mathrm{uv}}$ (Clontech, 8366-1)] was used as a fluorescent standard in quantification of GFP in potato with a BioRad VersaFluor ${ }^{\mathrm{TM}}$ Fluorometer with excitation $(390 \mathrm{~nm})$ and emission filters $(510 \mathrm{~nm})$.

\section{Results}

\subsection{Plastid transformation}

Experiments with pZS197 yielded 21 spectinomycin resistant $\left(\operatorname{spec}^{\mathrm{r}}\right)$ shoots from 179 bombarded plates (Table 1, Fig. 1A and B). Ten of these shoots were resistant to both spectinomycin and streptomycin (spectinomycin $300 \mathrm{mg} / \mathrm{L}$, streptomycin $300 \mathrm{mg} / \mathrm{L}$ ) (Fig. 1C and D). Nine $\operatorname{spec}^{\mathrm{r}}$ shoots were obtained from 103 shots with pMSK18, 4 of which were also streptomycin resistant (Table 1). Eight spect ${ }^{\mathrm{r}}$ shoots were obtained with pNtcZ7, but none of these were cross-resistant to streptomycin.

All $\operatorname{spec}^{\mathrm{r}}$ shoots were initially screened by PCR with primers for $a a d A$ or $a a d A$ and $g f p$ (data not shown). These confirmed that all spectinomycin plus streptomycin resistant lines contained aadA, while those with spectinomycin resistance alone did not and are believed to be spontaneous $\operatorname{spec}^{\mathrm{r}}$ mutants. PCR was also carried out with primers in the external (flanking) regions. PCR analysis of $5 \operatorname{spec}^{\mathrm{r}}$ pZS197 lines is shown in Fig. 2. Primers external to the vector sequence produced a wild type (WT) band of $2.8 \mathrm{kbp}$ in two of the $\operatorname{spec}^{\mathrm{r}}$ lines, which were streptomycin sensitive. The presence of a $4.1 \mathrm{kbp}$ band, and absence of the $2.8 \mathrm{kbp}$ band, for lines D-2, D-10 and D-13 indicates that these are homoplasmic plastid transformants. The same results were obtained with the remaining $\operatorname{spec}^{\mathrm{r}} / \mathrm{strep}^{\mathrm{r}}$ lines obtained with pZS197. Spectinomycin resistant lines were also analysed by Southern blotting with an rbcL probe (Fig. 2C). The probe hybridized to a $4.3 \mathrm{~kb}$ band in the plastid transformants and to a $3.0 \mathrm{~kb}$ band in the WT and $\operatorname{spec}^{\mathrm{r}}$ spontaneous mutants.

PCR analysis of shoots from experiments with the inverted repeat (IR) vector pMSK18 (Fig. 3B) clearly differentiated between the $1.3 \mathrm{~kb}$ WT product in control and $\operatorname{spec}^{\mathrm{r}}$ mutants (D-1, D-5 and D-7) and the $3.6 \mathrm{~kb}$ product from the transgenic lines (D-2, D-3, D-4, D-6). Thus, the number of double resistant $\left(\operatorname{spec}^{\mathrm{r}} / \mathrm{strep}^{\mathrm{r}}\right)$ shoots revealed in leaf strip assays exactly correlated with PCR and Southern data for both vectors.

Plants were grown to maturity in pots and compared to wild type Desiree plants, regenerated from untransformed leaf explants, and spontaneous spectinomycin resistant mutants recovered from biolistic experiments. No differences were detectable in the growth of the regenerated plants, or the yield of tubers (Fig. 4). Tuber explants
(A)

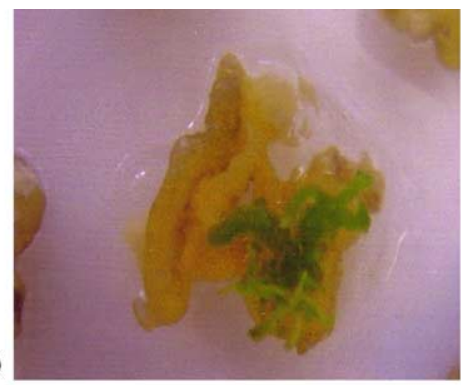

(C)

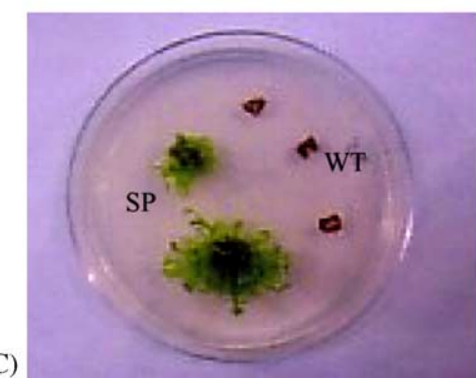

(B)

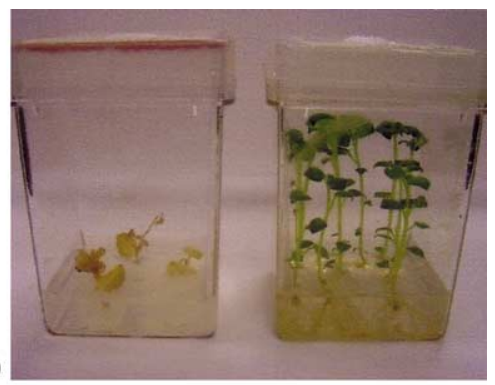

(D)

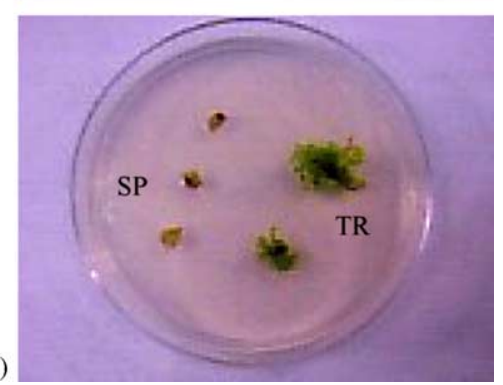

Fig. 1. Plastid transformation of potato using pZS197. (A) Primary selection of chloroplast transformants after incubation for 8 weeks, the leaf explants are bleached due to effective inhibition of plastid protein synthesis by spectinomycin. (B) Transplastomic plants on RM containing 400 mg/L spectinomycin. Wildtype control shoots (on left) are clearly spectinomycin sensitive. (C) Wild type (WT) and spontaneous mutants (SP) were differentiated by culture of leaves on SIM with spectinomycin. (D) Transplastomic plants (TR) were confirmed by resistance to both spectinomycin and streptomycin on SIM, whereas leaves of spontaneous mutants (SP) bleached on this medium. 


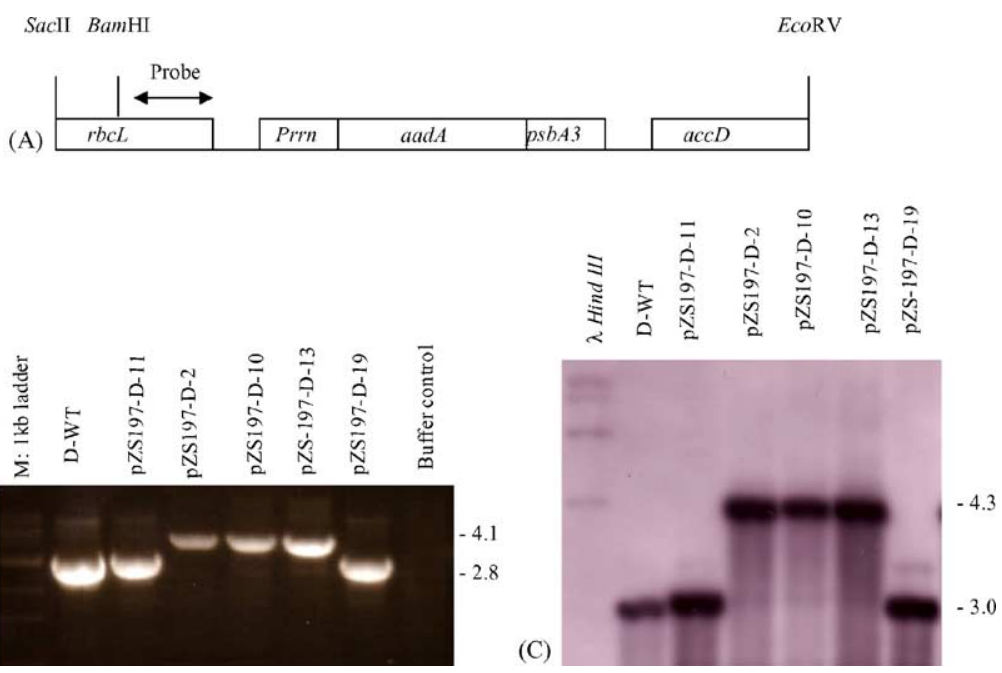

Fig. 2. Confirmation of integration of pZS197 DNA into the potato chloroplast genome. (A) Diagram of the target site of pZS197 in the potato plastid genome large single copy region. (B) Homoplasmic pZS197 lines identified with primers (RBCL and ACCD) external to the cloned ptDNA of the vector. Wild type or spontaneous $\operatorname{spec}^{r}$ mutants generated a $2.8 \mathrm{~kb}$ product, whereas a $4.1 \mathrm{~kb}$ product was amplified from transplastomic lines. (C) Southern blot of EcoRI/EcoRV digested DNA isolated from wild type and $\operatorname{spec}^{\mathrm{r}}$ shoots. Blot was probed with a labeled $550 \mathrm{bp} r b c L$ PCR product. The probe hybridized to a $3.0 \mathrm{~kb}$ band in WT and SP plants (pZS197-D11 and pZS197-D19) and a $4.3 \mathrm{~kb}$ transplastomic band was present in pZS197-D2, pZS197-D10 and pZS197-D13 homoplasmic transformants.

continued to exhibit high levels of spectinomycin resistance in shoot regeneration tests (Fig. 4C).

\subsection{Quantitative analysis of $G F P$}

GFP fluorescence was measured in two transplastomic lines (pMSK-D2 and pMSK-D3), one spontaneous mutant line (pMSK-D5), and wild type Desiree (Table 2). A low level of GFP activity was found in the leaves of the transplastomic lines, and an even lower level in the tubers. Faint GFP fluorescence in the leaf chloroplastscould also be detected visually by fluorescence microscopy (not shown). No GFP expression was observed from the spontaneous mutant (pMSK-D5) or wild type lines.

\section{Discussion}

For plastid transformation of potato we established efficient adventitious shoot regeneration from leaves, and used stringent antibiotic selection protocols. Plastid transformants were recovered with the two step regeneration protocol (CIM, SIM). This requirement for a callus phase for recovery of plastid transformation of potato is similar to that reported for tomato [9]. However, only 1 month on CIM was required for potato compared to about 6 months for tomato before shoot regeneration. In contrast, Sidorov et al. [7] reported potato plastid transformation with a single step protocol, but used a non-commercial genotype chosen on the basis of its responsiveness in vitro. We also found cutting leaves into small leaf explants $(3 \mathrm{~mm} \times 3 \mathrm{~mm})$ on selection media necessary for recovery of transformants as reported for tomato [9]. Sidorov et al. [7] obtained transformants with much larger $(5 \mathrm{~mm} \times 5 \mathrm{~mm})$ leaf explants of potato, but did not report if transformants were more efficiently recovered if smaller explants were used during the selection process.

Plastid transformation in potato was successful with two of the three vectors used. PCR and Southern blot analyses confirmed homoplasmy and incorporation of $\operatorname{aadA}$ (pZS197) into potato. There were insufficient sequence data for potato in the rps12-rrnl6 region to determine restriction sites to generate Southern blots of pMSK18 plants. However, PCR showed integration of aadA and $g f p$ in pMSK18 plants and the lack of WT PCR products in these plants indicates a high likelihood these are homoplasmic lines as well.

Sidorov et al. [7] reported 1 transformant per 15 shots for pZS197 and 1 per 35 shots for a vector targeting the rps 12rrn16 intergenic region of the IR. Our plastid transformation frequencies similarly varied with vectors targeting these two regions ( 1 per 18 shots for pZS197 and 1 per 25 shots for pMSK18). The flanking tobacco sequences in pZS197 are $98 \%$ identical to the potato chloroplast $a c c D-r b c L$ region. The rps12-rrn16 region is likely to have slightly lower sequence similarity between potato and tobacco than the $r b c L-a c c D$ region, but it is not known if this is the reason for the lower transformation frequency obtained in both these studies for tobacco vectors targeting the IR in potato compared to the LSC. It is notable that with both vectors a similar proportion, roughly half, of the spectinomycin resistant shoots selected were plastid transformants, the remainder presumed to be spontaneous mutants. In contrast, Sidorov et al. [7] report three times as many spontaneous mutants as transformants.

Plastid transformation in tobacco [19] and tomato (Nugent et al., submitted) is known to occur by multiple 
(A)

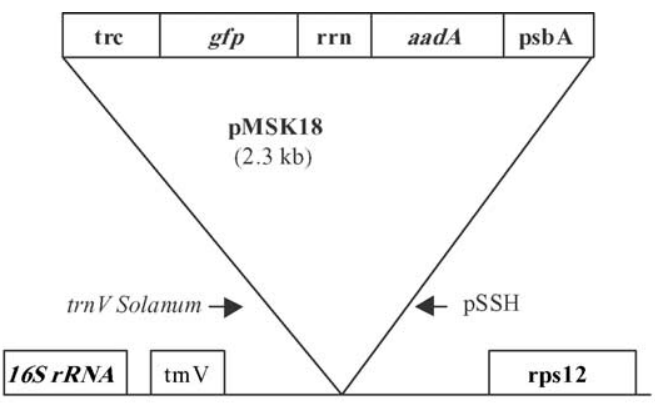

(B)

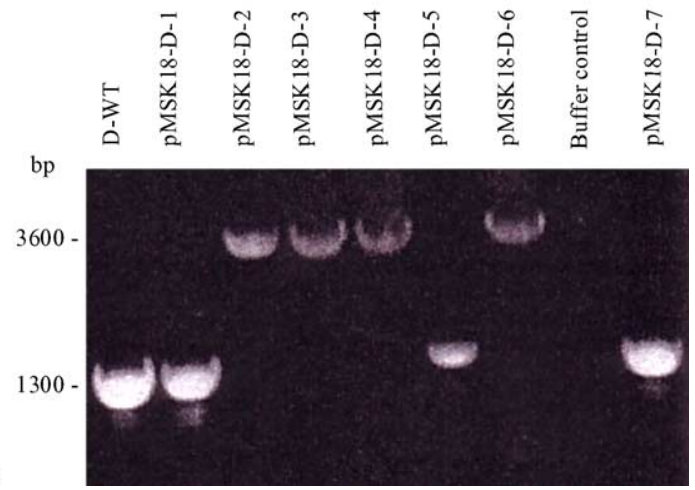

Fig. 3. PCR analysis of spectinomycin resistant pMSK18 plants. (A) Diagram of the site of integration of the expression cassette in the rps12-16SrRNA intergenic region of the inverted repeat (IR). The primer pair pSSH-rev/trnV Solanum flanking the expression cassette insertion site in the potato chloroplast genome are shown. (B) The $3.6 \mathrm{~kb}$ band confirms correct integration of aadA and $g f p$ into the chloroplast genome of homoplasmic lines pMSK18-D2, pMSK18-D3, pMSK18-D4 and pMSK18-D6. The $1.3 \mathrm{~kb}$ WT band is present in spontaneous mutant lines pMSK18-D1, pMSK18-D5 and pMSK18-D7.

recombination events in the rps12-rrn16 region when a vector containing homeologous cloned Solanum plastid DNA was used. Multiple recombination events may have occurred in this region in potato plastid transformants with
Table 2

GFP expression levels in transplastomic pMSK18 lines

\begin{tabular}{lll}
\hline Plant line & \multicolumn{2}{l}{$\begin{array}{l}\text { GFP concentration } \\
(\mu \mathrm{g} / \mathrm{mg} \text { of exactable protein })\end{array}$} \\
\cline { 2 - 3 } & Leaves & Tubers \\
\hline Desiree-WT & 0 & 0 \\
pMSK18-D2 & 0.175 & 0.035 \\
pMSK18-D3 $^{\text {pMSK18-D5 }}$ & 0.170 & 0.020 \\
\hline
\end{tabular}

${ }^{a}$ Spontaneous mutant line.

these homeologous tobacco vectors, however, neither our study nor that of Sidorov et al. [7] included the sequence analysis needed to examine this possibility.

Potato and tomato [9] plastid transformants are generated at 10-30 times lower frequencies than tobacco. This frequency is close to the low frequency obtained in Arabidopsis [6] and Lesquerella [11]. This compares with one plastid transformant per bombarded sample for tobacco [5]. The generation of species specific vectors may not be necessary in all cases [11]. However, it remains to be tested if a higher transformation frequency could be obtained in potato and other species outside the Nicotiana genus with a species specific vector compared to one with identically sized flanking regions in a partially homologous vector.

The two tobacco rps12-rrnl6 vectors in this study contain the same tobacco flanking regions [12]. The lack of potato plastid transformants with pNtcZ7 is probably due to the trc promoter driving aadA expression, compared to Prrn in pMSK18. This bacterial promoter can give sufficient expression of the aadA gene to select plastid transformants in tobacco. However, transformants were generated at 10-20 times lower frequency with a vector with $\operatorname{trc}$-aadA [20] compared to Prrn-aadA [5]. This emphasizes the importance of using a strong plastid promoter for crops with a less
(A)

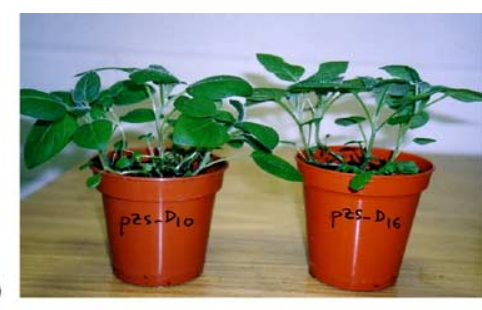

(B)

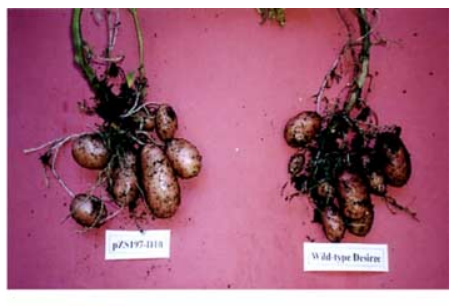

(C)

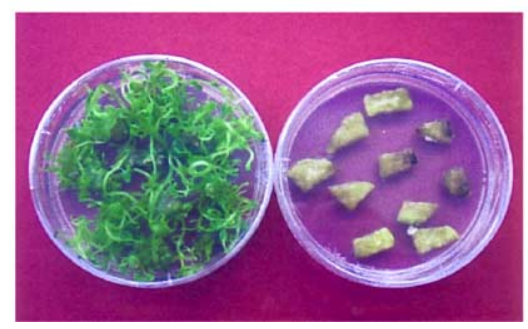

Fig. 4. Transplastomic plants and tubers. (A) Transplastomic line pZS197-D10 (left) and spontaneous mutant line pZS197-D16 (right) after 4 weeks in soil. (B) Tubers from transplastomic line pZS197-D10 (left) and wild type cv. Desiree, regenerated from culture (right), harvested after 14 weeks in soil in growth room at $22{ }^{\circ} \mathrm{C}$. (C) Spectinomycin resistant test on tuber segments. Pieces from tubers illustrated in 4(B) were placed on CIM medium for 4 weeks, then transferred to SIM medium containing $300 \mathrm{mg} / \mathrm{L}$ spectinomycin. Dishes were photographed after 6 weeks on the latter medium, pZS197-D10 (left) and wild type cv. Desiree (right). 
optimal regenerative capacity. We predict a theoretical minimum of 250 bombardments of potato would be needed to capture a plastid transformant with a $t r c$-aadA vector in potato, given the lower efficiency of trc in plastids and the lower transformation frequency of potato.

GFP concentration in transplastomic lines was not as high as reported previously for $g f p$ constructs under the control of Prrn in transplastomic potato [7] and tobacco [21]. This result was similar to that reported of tobacco using the bacterial trc promoter further supporting the lower efficacy of this prokaryotic promoter. Transplastomic plants expressing $g f p$ from the $r r n$ promoter contained approximately 90 fold more GFP than plants using psbA or trc promoters [22]. In view of the low level of GFP expression in the leaves, and the fact that very poor expression would be expected in tubers, it is perhaps surprising that GFP from the latter could be detected. In fact tuber expression was $12-20 \%$ of that in the leaves, whereas previously only $1 \%$ of the leaf value was found in tubers, when $g f p$ expression was driven by the $r r n$ promoter.

We have confirmed that plastid transformation is reproducible in a commercial cultivar of this important crop plant and we are currently generating potato plastid transformants containing genes for agronomically important traits.

\section{Acknowledgments}

We thank Dr. Eugene Kane for useful discussions, Prof. Pal Maliga, Rutgers University, for providing pZS197 and Prof. John C. Gray, Cambridge University, for pMSK18 and pNtcZ7. This research was supported by funds from an Irish Government Higher Education Authority grant (PRTL), and the European Union (EU) 5th Framework programme (Grant: QLK-CT-1999-00692).

\section{References}

[1] P. Maliga, Towards plastid transformation in flowering plants, Trends Biotech. 11 (1993) 101-107.

[2] R. Bock, Transgenic plastids in basic research and plant biotechnology, J. Mol. Biol. 312 (2001) 425-438.

[3] H. Daniell, R. Datta, S. Varma, S. Gray, S.B. Lee, Containment of herbicide resistance through genetic engineering of the chloroplast genome, Nat. Biotechnol. 16 (1998) 345-348.
[4] Z. Svab, P. Hajdukiewicz, P. Maliga, Stable transformation of plastids in higher plants, Proc. Natl. Acad. Sci. U.S.A. 87 (1990) 8526-8530.

[5] Z. Svab, P. Maliga, High-frequency plastid transformation in tobacco by selection for a chimeric aadA gene, Proc. Natl. Acad. Sci. U.S.A. 90 (1993) 913-917.

[6] S.R. Sikdar, G. Serino, S. Chaudhuri, P. Maliga, Plastid transformation in Arabidopsis thaliana, Plant Cell Rep. 18 (1998) 20-24.

[7] V.A. Sidorov, D. Kasten, S.Z. Pang, P.T.J. Hajdukiewicz, J.M. Staub, N.S. Nehra, Stable chloroplast transformation in potato: use of green fluorescent protein as a plastid marker, Plant J. 19 (1999) 209-216.

[8] M.S. Khan, P. Maliga, Fluorescent antibiotic resistance marker for tracking Plastid transformation in higher plants, Nat. Biotechnol. 17 (1999) 910-915.

[9] S. Ruf, M. Hermann, I.J. Berger, H. Carrer, R. Bock, Stable genetic transformation of tomato plastids: foreign protein expression in fruit, Nat. Biotechnol. 19 (2001) 870-875.

[10] B.K. Hou, Y.H. Zhou, L.H. Wan, Z.L. Zhang, G.F. Shen, Z.H. Chen, Z.M. Hu, Chloroplast transformation in oilseed rape, Transgenic Res. 12 (2003) 111-114.

[11] M. Skarjinskaia, Z. Svab, P. Maliga, Plastid transformation in Lesquerella fendleri, an oilseed Brassicacea, Transgenic Res. 12 (2003) 115-122.

[12] J.M. Hibberd, P.J. Linley, M.S. Khan, J.C. Gray, Transient expression of green fluorescent protein in various plastid types following microprojectile bombardment, Plant J. 16 (1998) 627-632.

[13] T. Murashige, F. Skoog, A revised medium for the growth and bioassay with tobacco tissue culture, Physiol. Plant 15 (1962) 473-497.

[14] O.L. Gamborg, R.A. Muller, K. Ojima, Nutrient requirements of suspension culture of soybean root cells, Exp. Cell Res. 50 (1968) $151-158$

[15] A. Beaujean, R.S. Sangwan, A. Lecardonnel, B.S. Sangwan-Norreel, Agrobacterium-mediated transformation of three economically important potato cultivars using sliced internodal explants: an efficient protocol of transformation, J. Exp. Bot. 49 (1998) 1589-1595.

[16] J.E. Frey, Genetic flexibility of plant chloroplasts, Nature 398 (1999) $115-116$

[17] M.S. McCabe, J.B. Power, A.M.M. De Laat, M.R. Davey, Detection of single-copy genes in DNA from transgenic plants by nonradioactive Southern blot analysis, Mol. Biotechnol. 7 (1997) 79-84.

[18] T. Remans, P.M. Schenk, J.M. Manners, C.P.L. Grof, A.R. Elliott, A protocol for the fluorometric quantification of mGFP5-ER and sGFP (S65T) in transgenic plants, Plant Mol. Biol. Rep. 17 (1999) 385-395.

[19] T.A. Kavanagh, N.D. Thanh, N.T. Lao, N. McGrath, S.O. Peter, E.M. Horvath, P.J. Dix, P. Medgyesy, Homologous plastid DNA transformation in tobacco is mediated by multiple recombination events, Genetics 152 (1999) 1111-1122.

[20] P. Mäenpää, E.B. Gonzaler, L. Chen, M.S. Khan, J.C. Gray, E.M. Aro, The $y c f$ (orf62) gene in plant chloroplast genome encodes a hydrophobic protein of stromal thylakoid membranes, J. Exp. Bot. 51 (2000) 375-382.

[21] M.L. Reed, S.K. Wilson, C.A. Sutton, M.R. Hanson, High level expression of a synthetic red-shifted GFP coding region incorporated into transgenic chloroplasts, Plant J. 27 (2001) 257-265.

[22] C.A. Newell, I. Birch-Machin, J.H. Hibberd, J.C. Gray, Expression of green fluorescent protein from bacterial and plastid promoters in tobacco chloroplasts, Transgenic Res. 12 (2003) 631-634. 